\title{
Circular Samples as Objects for Magnetic Resonance Imaging - Mathematical Simulation, Experimental Results
}

\author{
Ivan Frollo, Andrej Krafčík, Peter Andris, Jiř́i Přibil, Tomáš Dermek \\ Institute of Measurement Science, Slovak Academy of Sciences, Bratislava, Slovakia, frollo@savba.sk
}

\begin{abstract}
Circular samples are the frequent objects of "in-vitro" investigation using imaging method based on magnetic resonance principles. The goal of our investigation is imaging of thin planar layers without using the slide selection procedure, thus only $2 \mathrm{D}$ imaging or imaging of selected layers of samples in circular vessels, eppendorf tubes,.. compulsorily using procedure "slide selection". In spite of that the standard imaging methods was used, some specificity arise when mathematical modeling of these procedure is introduced. In the paper several mathematical models were presented that were compared with real experimental results. Circular magnetic samples were placed into the homogenous magnetic field of a low field imager based on nuclear magnetic resonance. For experimental verification an MRI 0.178 Tesla ESAOTE Opera imager was used.
\end{abstract}

Keywords: Circular physical objects, mathematical modeling, magnetic susceptibility, Magnetic Resonance Imaging.

\section{INTRODUCTION}

$\mathrm{I}_{\mathrm{s}}^{\mathrm{N}}$ MAGING METHODS used for biological and physical structure investigation, based on Nuclear Magnetic Resonance (NMR), have become a regular diagnostic procedure. Specific occurrence is observed when an object, consisting of a soft magnetic material, is inserted into a static homogeneous magnetic field. This results in small variations of the static homogeneous magnetic field near the sample. The acquired image represents a modulation of the basic homogeneous magnetic field of the imager detectable by gradient-echo (GRE) or spin-echo (SE) imaging sequences.

First attempts of a direct measurement of the magnetic field variations created in living and physical tissues by a simple wire fed by a current were published in [1]. A method utilizing the divergence in gradient strength that occurs in the vicinity of a thin current-carrying copper wire was introduced in [2]. A simple experiment with thin, pulsed electrical current-carrying wire and imaging of a magnetic field, using a plastic sphere filled with agarose gel as phantom, was published in [3]. Comparison of traditional segmentation methods with 2D active contour methods was discussed in [4]. Single biogenic soft magnetite nanoparticle physical characteristics in biological objects were introduced in [5]. Magnetic resonance imaging of the static magnetic field distortion caused by magnetic nanoparticles: Simulation and experimental verification were published in [6].

In the paper several mathematical models are presented, that were compared with real experimental results. Circular magnetic samples were placed into the homogenous magnetic field of a low field imager. Magnetic resonance imaging method used for soft magnetic material detection, computation of the magnetic field variations based on theory of magnetism and comparison of theoretical results with experimental images are the main goal of the next text. Via experiments we want to prove that the proposed method is perspective for soft magnetic materials testing using magnetic resonance imaging methods.

\section{SUBJECT \& METHODS}

In our experiments a soft ferromagnetic or paramagnetic object was placed into the homogeneous magnetic field of an MRI imager. The homogeneous magnetic field near the sample is deformed. For simplicity and easy experimental verification a circular object was selected and theoretically analyzed.

\section{A. Circular sample - theoretical principles}

Let us suppose that the soft magnetic planar layer is positioned in the $x-y$ plane of the rectangular coordinate system and the thickness of the layer is neglected. According to Fig.1., we suppose the circular layers is limited by diameter $\mathrm{R}$, ( $\mathrm{R}^{\prime}$ for annulus). Let us suppose that $\mathrm{dS}[\mathrm{x}, \mathrm{y}]$ is an elementary surface element. The static magnetic field $\mathrm{B}_{0}$ of the MR imager is parallel with the $\mathrm{z}$ axis. The task is to calculate the $B_{z}$ component of the magnetic field in the point $\mathrm{A}\left[\mathrm{x}_{0}, \mathrm{y}_{0}, \mathrm{z}_{0}\right]$.

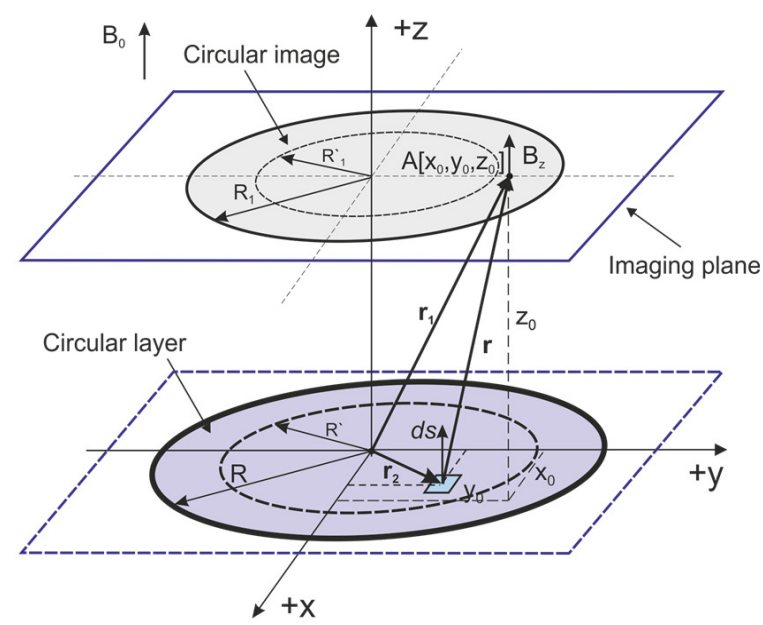

Fig.1. Fundamental configuration of the circular planar magnetic layer. The sample is positioned in $x-y$ plane of the rectangular coordinate system. The thickness of the layers is neglected. 
For theoretical analysis it is possible to consider the magnetic double layer as magnetic dipoles continuously distributed in the surface of the layer S. Then the overall magnetic moment $\mathrm{M}$ relating to the surface $\mathrm{S}$ could be expressed by a surface integral:

$$
\boldsymbol{m}_{\mathrm{s}}=\int_{\mathrm{S}} \boldsymbol{M}_{\mathrm{S}}(\boldsymbol{r}) \mathrm{dS} \quad \text { and } \quad \boldsymbol{M}_{S}=I \boldsymbol{n}
$$

where: $\boldsymbol{m}_{\mathrm{s}}$ - is magnetic dipole moment in a particular point, $\boldsymbol{r}$ - is a position vector, I - is a current equivalent to planar density of dipole moment of the magnetic double layer, and $\mathbf{n}$ - is a unit normal vector of surface $\mathrm{S}$ in a particular point.

Magnetic double layer is considered having a homogeneous density of the dipole moment that is oriented in every point the direction of surface normal vector perpendicular to the layer surface. Bearing in mind the superposition principle it is possible to express the vector potential of the double layer in the shape of surface integral:

$$
\mathbf{A}(\mathbf{r})=\frac{\mu_{0} I}{4 \pi} \int_{S} \frac{\mathrm{d} \mathbf{S} \times \mathbf{r}}{r^{3}}=\frac{\mu_{0} I}{4 \pi} \int_{S} \operatorname{grad}\left(\frac{1}{r}\right) \times \mathrm{d} \mathbf{S}
$$

After using general formula for vector potential and magnetic induction $\mathbf{B}=\operatorname{curl} \mathbf{A}$, we get the final formula for magnetic induction of the magnetic double layer and applicable also for the close current loop calculation as follows:

$$
\mathbf{B} \equiv \operatorname{curl} \mathbf{A}=\frac{\mu_{0} I}{4 \pi} \int_{S}\left(\frac{3 \mathbf{r}(\mathrm{d} \mathbf{S} \cdot \mathbf{r})}{r^{5}}-\frac{\mathrm{d} \mathbf{S}}{r^{3}}\right),
$$

where the position vectors according to the Fig.1. can be exppressed as:

$$
\begin{aligned}
& \mathbf{r}=\mathbf{r}_{1}-\mathbf{r}_{2} \text { and } \\
& \mathrm{r}=\sqrt{\left(\mathrm{x}_{0}-\mathrm{x}\right)^{2}+\left(\mathrm{y}_{0}-\mathrm{y}\right)^{2}+\left(\mathrm{z}_{0}-\mathrm{z}\right)^{2}} .
\end{aligned}
$$

If we consider single circular sample with normal vector $\mathbf{n}=[0,0,1]$, equation (3) can be explicitly rewriten to the form

$$
\begin{aligned}
& B_{x}\left(x_{0}, y_{0}, z_{0}\right)=\frac{\mu_{0} I}{4 \pi} \int_{S}\left(\frac{3\left(x_{0}-x\right)\left(z_{0}-z\right)}{r^{5}}\right) \mathrm{d} x \mathrm{~d} y \\
& B_{y}\left(x_{0}, y_{0}, z_{0}\right)=\frac{\mu_{0} I}{4 \pi} \int_{S}\left(\frac{3\left(y_{0}-y\right)\left(z_{0}-z\right)}{r^{5}}\right) \mathrm{d} x \mathrm{~d} y \\
& B_{z}\left(x_{0}, y_{0}, z_{0}\right)=\frac{\mu_{0} I}{4 \pi} \int_{S}\left(\frac{3\left(z_{0}-z\right)^{2}}{r^{5}}-\frac{1}{r^{3}}\right) \mathrm{d} x \mathrm{~d} y
\end{aligned}
$$

Direct evaluation of Eq. 3. is quite problematic. Some problems can be solved analytically in other way, directly from Maxwell equations. But still, almost everything is solvable numerically, although approximatelly.

\section{B. Calculation using analytical solution}

According to Fig.2., we suppose three circular concentric vessels. Relative permeabilities: $\mu_{1}, \mu_{2}$, environment permeability: $\mu_{0}$.

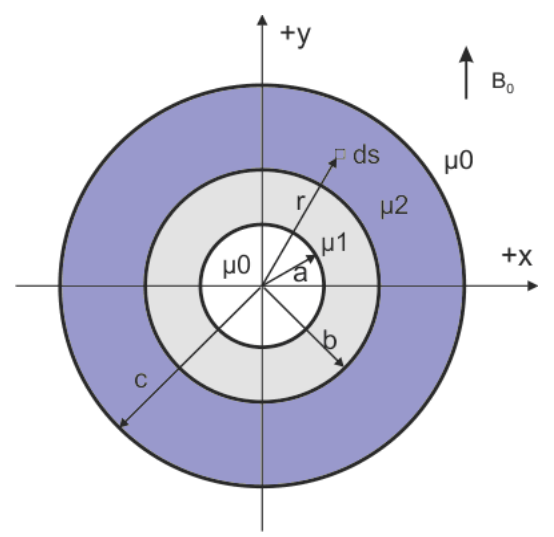

Fig.2. Basic configuration of the magnetic planar layer circular sample positioned in $\mathrm{x}-\mathrm{y}$ plane of the rectangular coordinate system.

The radial distance of a position vector in cylindrical coordinate system can be expressed as:

$$
\rho \equiv \sqrt{x^{2}+y^{2}} .
$$

Because of the symmetry of configuration, Laplace equation can be expressed in cylindrical coordinate system as

$$
\begin{aligned}
& \nabla^{2} A(\rho, \varphi, z) \equiv \\
& \equiv \frac{\partial^{2} A}{\partial \rho^{2}}+\frac{1}{\rho} \frac{\partial A}{\partial \rho}+\frac{1}{\rho^{2}} \frac{\partial^{2} A}{\partial \varphi^{2}}+\frac{\partial^{2} A}{\partial z^{2}}=0,
\end{aligned}
$$

and solved analytically by separation of variables. For nonzero $z$-component of vector potential in $k$-th cylindrical layer or environment we can write [10]:

$$
A_{\mathrm{k}}=\left(C_{\mathrm{k}} \rho+\frac{D_{\mathrm{k}}}{\rho}\right) \cos \varphi,
$$

what yields radial and tangential components of magnetic flux density in the form:

$$
\begin{aligned}
& B_{\rho}^{\mathrm{k}}=\frac{1}{\rho} \frac{\partial A_{\mathrm{k}}}{\partial \varphi}=-\left(C_{\mathrm{k}}+\frac{D_{\mathrm{k}}}{\rho^{2}}\right) \sin \varphi, \\
& B_{\varphi}^{\mathrm{k}}=-\frac{\partial A_{\mathrm{k}}}{\partial \rho}=-\left(C_{\mathrm{k}}-\frac{D_{\mathrm{k}}}{\rho^{2}}\right) \cos \varphi,
\end{aligned}
$$

where $C_{\mathrm{k}}$ and $D_{\mathrm{k}}$ are constants calculated for every region (domain). In material domain where $\rho=0$, vector potential 
has to be finite, what is fulfilled only if $\left.D\right|_{\rho=0}=0$. In a similar way, for domain where $\rho \rightarrow \infty\left(\mu=\mu_{0}\right)$, magnetic field has the intensity same as original external homogeneous magnetic field, therefore $\left.C\right|_{\rho \rightarrow \infty}=\mu_{0} H_{0}$.

Remaining constants can be determined from boundary conditions on each cylindrical interface between two material domains (e.g. I and II with radius $R_{I}$ ), rewritten explicitly in the form:

$$
\begin{gathered}
\left.B_{\rho}^{\mathrm{I}}\right|_{\rho=R_{\mathrm{I}}}=\left.B_{\rho}^{\mathrm{II}}\right|_{\rho=R_{\mathrm{I}}}, \\
\left.\frac{1}{\mu_{\mathrm{I}}} B_{\varphi}^{\mathrm{I}}\right|_{\rho=R_{\mathrm{I}}}=\left.\frac{1}{\mu_{\mathrm{II}}} B_{\varphi}^{\mathrm{II}}\right|_{\rho=R_{\mathrm{I}}},
\end{gathered}
$$

\section{Calculation example of 1 cylinder in $\boldsymbol{H}_{0}$}

For one magnetic cylinder with axis identical with z-axis of Cartesian coordinate system, whose radius is R, Fig.2., and permeability is $\mu_{1}$, located in surrounding environment with permeability $\mu_{2}=\mu_{0} \equiv 4 \pi \times 10^{-7} \mathrm{~N} / \mathrm{A}^{2}$ and magnetic field intensity $\mathbf{H}_{0}=\left[0, \mathrm{H}_{0}, 0\right]$, magnetic vector potential (8) can be calculated for $\mathrm{k}$-th medium domain directly by solving system of linear equations, derived from boundary conditions (10), expressed in matrix notation as:

$$
\left(\begin{array}{cccc}
1 & \frac{1}{R_{1}^{2}} & -1 & -\frac{1}{R_{1}^{2}} \\
\frac{1}{\mu_{1}} & -\frac{1}{\mu_{1} R_{1}^{2}} & -\frac{1}{\mu_{2}} & \frac{1}{\mu_{2} R_{1}^{2}} \\
0 & 1 & 0 & 0 \\
0 & 0 & \frac{1}{\mu_{2}} & 0
\end{array}\right) \cdot\left(\begin{array}{l}
C_{1} \\
D_{1} \\
C_{2} \\
D_{2}
\end{array}\right)=\left(\begin{array}{c}
0 \\
0 \\
0 \\
H_{0}
\end{array}\right)
$$

Solution of this system of linear equations is shown in particular case for $\mathrm{R}=\mathrm{a}$, and $\mu_{1}, \mu_{2}=\mu_{0}$.

$$
\begin{aligned}
& \mathrm{C}_{1}=\frac{2 \mathrm{H}_{0} \mu_{0} \mu_{1}}{\mu_{0}+\mu_{1}} \\
& \mathrm{D}_{1}=0 \\
& \mathrm{C}_{2}=\mathrm{H}_{0} \mu_{0} \\
& \mathrm{D}_{2}=-\frac{\mathrm{H}_{0} \mathrm{a}^{2} \mu_{0}\left(\mu_{0}-\mu_{1}\right)}{\mu_{0}+\mu_{1}}
\end{aligned}
$$

Calculation results are depicted in Fig.3. The pictures illustrates the magnetic induction distribution of originally static homogeneous magnetic field $\mathbf{H}_{0}=\mu_{0} \mathbf{B}_{0}, \mathbf{B}_{0}=0.178 \mathrm{~T}$ affected by one magnetic cylinder. The environment is declared with permeability $\mu_{2}=\mu_{0}$. The range of calculated values is: $0.12-0.24$ Tesla. The values above the middle value $0.178 \mathrm{~T}$ are typical in NMR images of circular samples after application of GRE measuring sequence. The values below the middle value are responsible for border phenomena in the final images.

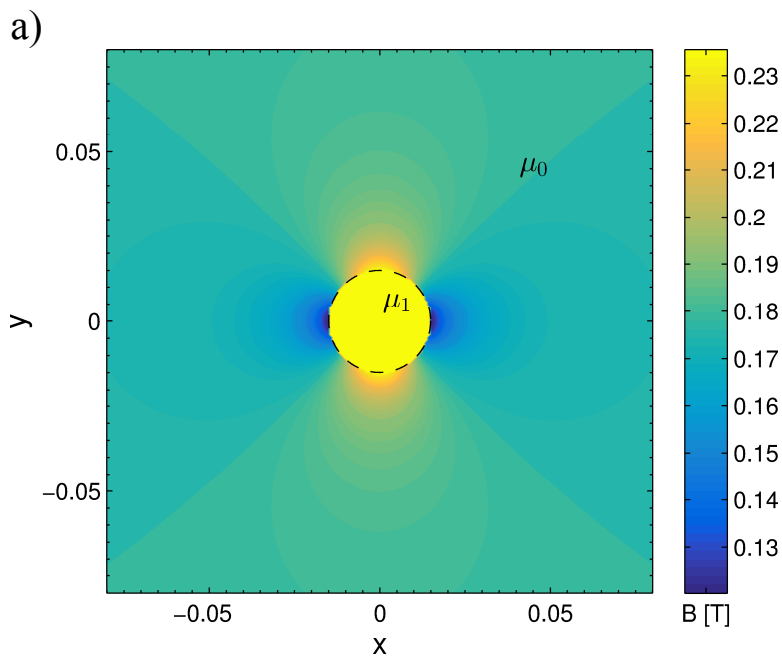

b)

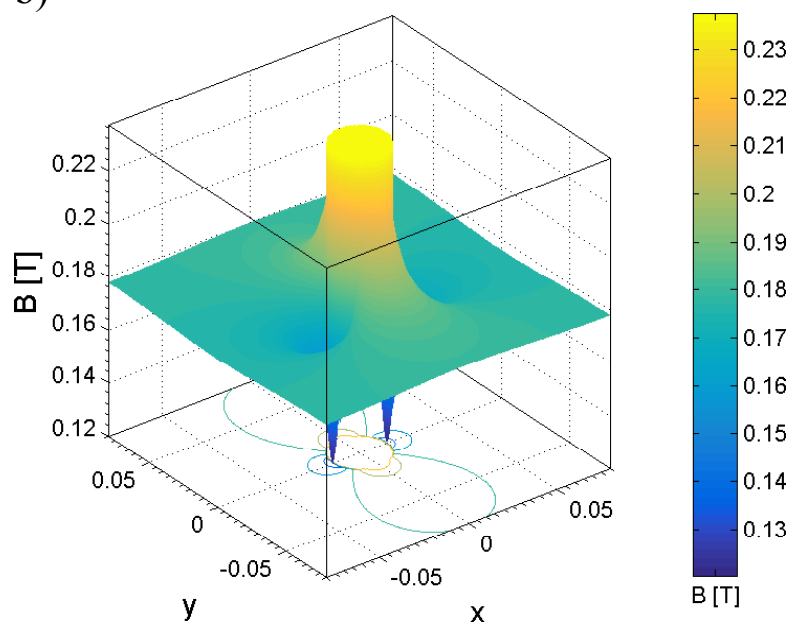

Fig.3. Magnetic induction distribution of originally homogeneous magnetic field $\mathbf{H}_{0}=\mu_{0} \mathrm{~B}_{0}, \mathbf{B}_{0}=0.178 \mathrm{~T}$ affected by one magnetic cylinder with permeability: $\mu_{1}=2 \mu_{0}$ in environment with permeability $\mu_{2}=\mu_{0} \equiv 4 \pi \times 10^{-7} \mathrm{~N} / \mathrm{A}^{2}$.

\section{Calculation example of 3 cylinders in $\boldsymbol{H}_{0}$}

For three concentric magnetic cylinders with axis identical with $\mathrm{z}$-axis of Cartesian coordinate system, whose radii comply $\mathrm{R} 1<\mathrm{R} 2<\mathrm{R} 3$ and permeabilities are $\mu_{1}, \mu_{2}, \mu_{3}$ located in surrounding environment with permeability $\mu_{4}$ and magnetic field intensity $\mathbf{H}_{0}=\left[0, H_{0}, 0\right]$, magnetic vector potential (8) can be calculated for $\mathrm{k}$-th medium domain directly by solving system of linear equations, derived from boundary conditions (10), expressed in matrix notation as

$\left(\begin{array}{cccccccc}1 & \frac{1}{R_{1}^{2}} & -1 & -\frac{1}{R_{1}^{2}} & 0 & 0 & 0 & 0 \\ \frac{1}{\mu_{1}} & -\frac{1}{\mu_{1} R_{1}^{2}} & -\frac{1}{\mu_{2}} & \frac{1}{\mu_{2} R_{1}^{2}} & 0 & 0 & 0 & 0 \\ 0 & 0 & 1 & \frac{1}{R_{2}^{2}} & -1 & -\frac{1}{R_{2}^{2}} & 0 & 0 \\ 0 & 0 & \frac{1}{\mu_{2}} & -\frac{1}{\mu_{2} R_{2}^{2}} & -\frac{1}{\mu_{3}} & \frac{1}{\mu_{3} R_{2}^{2}} & 0 & 0 \\ 0 & 0 & 0 & 0 & 1 & \frac{1}{R_{3}^{2}} & -1 & -\frac{1}{R_{3}^{2}} \\ 0 & 0 & 0 & 0 & \frac{1}{\mu_{3}} & -\frac{1}{\mu_{3} R_{3}^{2}} & -\frac{1}{\mu_{4}} & \frac{1}{\mu_{4} R_{3}^{2}} \\ 0 & 1 & 0 & 0 & 0 & 0 & 0 & 0 \\ 0 & 0 & 0 & 0 & 0 & 0 & \frac{1}{\mu_{4}} & 0\end{array}\right) \cdot\left(\begin{array}{c}C_{1} \\ D_{1} \\ C_{2} \\ D_{2} \\ C_{3} \\ D_{3} \\ C_{4} \\ D_{4}\end{array}\right)=\left(\begin{array}{c}0 \\ 0 \\ 0 \\ 0 \\ 0 \\ 0 \\ 0 \\ H_{0}\end{array}\right)$


Solution of this system of linear equations is shown in particular case, for:

$\mathrm{R}_{1}<\mathrm{R}_{2}<\mathrm{R}_{3}$, where $\mathrm{R}_{1}=a, \mathrm{R}_{2}=b, \mathrm{R}_{3}=c$.

$\mu_{1}=\mu_{0}, \mu_{2}=2 \mu_{0}, \mu_{3}=3 \mu_{0}, \mu_{4}=\mu_{0}$,

and $\mu_{0}=4 \pi \times 10^{-7} \mathrm{~N} / \mathrm{A}^{2}$ is vacuum permeability.

$$
\begin{aligned}
& C_{\text {I }}=-H_{0} \frac{24 b^{2} c^{2} \mu_{0}}{5 a^{2} b^{2}-2 a^{2} c^{2}+3 b^{4}-30 b^{2} c^{2}}, \\
& D_{\mathrm{I}}=0, \\
& C_{\mathrm{II}}=-H_{0} \frac{36 b^{2} c^{2} \mu_{0}}{5 a^{2} b^{2}-2 a^{2} c^{2}+3 b^{4}-30 b^{2} c^{2}}, \\
& D_{\mathrm{II}}=H_{0} \frac{12 a^{2} b^{2} c^{2} \mu_{0}}{5 a^{2} b^{2}-2 a^{2} c^{2}+3 b^{4}-30 b^{2} c^{2}}, \\
& C_{\mathrm{III}}=-H_{0} \frac{3 c^{2} \mu_{0}\left(a^{2}+15 b^{2}\right)}{5 a^{2} b^{2}-2 a^{2} c^{2}+3 b^{4}-30 b^{2} c^{2}}, \\
& D_{\mathrm{III}}=H_{0} \frac{3 c^{2} \mu_{0}\left(5 a^{2} b^{2}+3 b^{4}\right)}{5 a^{2} b^{2}-2 a^{2} c^{2}+3 b^{4}-30 b^{2} c^{2}}, \\
& C_{\mathrm{IV}}=\mu_{0} H_{0}, \\
& D_{\mathrm{IV}}=H_{0} \frac{c^{2} \mu_{0}\left(10 a^{2} b^{2}-a^{2} c^{2}+6 b^{4}-15 b^{2} c^{2}\right)}{5 a^{2} b^{2}-2 a^{2} c^{2}+3 b^{4}-30 b^{2} c^{2}} .
\end{aligned}
$$

Resultant $z$-component of vector potential respecting four regions using constants $C_{\mathrm{I}}$ to $C_{\mathrm{IV}}$ and $D_{\mathrm{I}}$ to $D_{\mathrm{IV}}$ in planar approximation is as follows:

$$
A= \begin{cases}A_{\mathrm{I}}, & \text { for } \quad \rho \leq \mathrm{a}, \\ A_{\mathrm{II}}, & \text { for } a<\rho \leq \mathrm{b}, \\ A_{\mathrm{III}}, & \text { for } b<\rho \leq \mathrm{c}, \\ A_{\mathrm{IV}}, & \text { for } \mathrm{c}<\rho,\end{cases}
$$

and vector potential $\mathbf{A}=[0,0, A]$. Resultant flux density of magnetic field will be:

$$
\mathbf{B}=\nabla \times \mathbf{A} .
$$

Its magnetic flux density lines is possible to draw as a stream plot, relative values:

StreamPlot $\left[\left\{\mathrm{H}_{\mathrm{r}}\right\},\{\mathrm{x},-0.08,0.08\},\{\mathrm{y},-0.08,0.08\}\right]$.

Mathematical model of the distribution of homogeneous magnetic field $\mathrm{H}_{0}$, lines of force affected by 3 concentric cylinders is depicted in Fig 4.

Calculation results of magnetic induction distribution of stationary homogeneous magnetic field $\mathbf{H}_{0}$ affected by 3 concentric magnetic cylinders are depicted in Fig.5. These are theoretical examples using relative values of magnetic permeabilities. The range of calculated values: $0.15-0.29 \mathrm{~T}$. The values above and below the middle value $0.178 \mathrm{~T}$ are typical in NMR images of circular samples after application of GRE measuring sequence. The permeability values are relative. For simulation of real samples it is necessary to use the actual values.

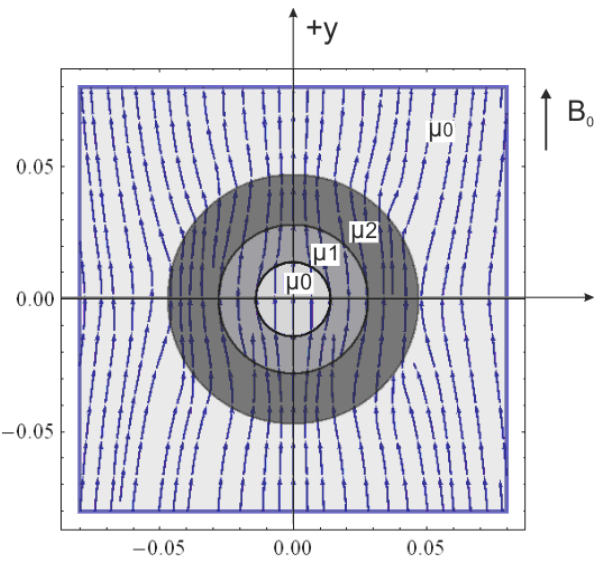

Fig.4. Mathematical model of the distribution of homogeneous magnetic field $\mathrm{H}_{0}$, lines of force affected by 2 concentric cylinders with relative permeabilities: $\mu_{1}=2$ and $\mu_{2}=3, \mu_{0}$ is an environment permeability.

a)

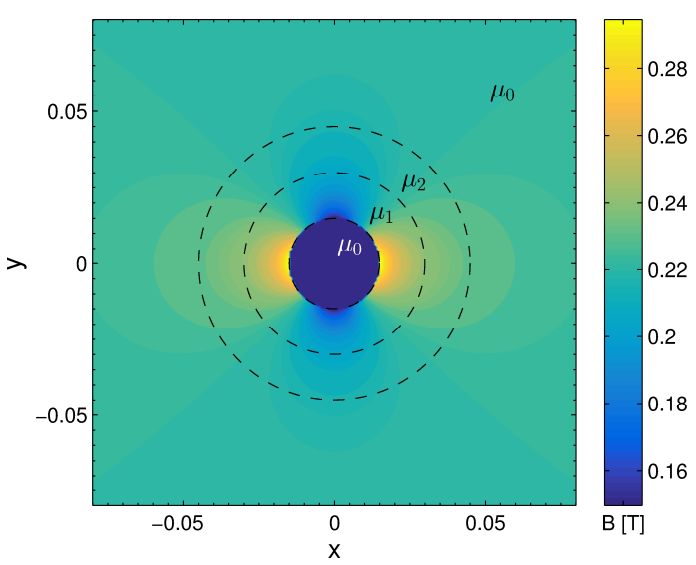

b)

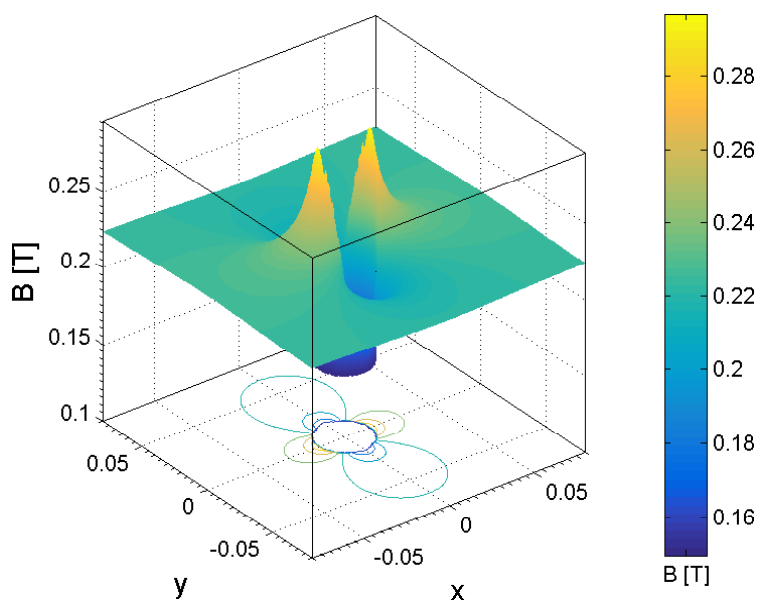

Fig.5. Magnetic induction distribution of static homogeneous magnetic field $\mathbf{H}_{0}$ affected by 3 concentric magnetic cylinders with permeabilities: $\mu_{1}=\mu_{0}, \mu_{2}=2 \mu_{0}, \mu_{3}=3 \mu_{0}$ in environment with permeability $\mu_{4}=\mu_{0}$.

\section{Experimental results}

For experimental evaluation of the mathematical modeling we have chosen two simple laboratory arrangements for application of magnetic resonance imaging methods. 


\section{Liquid circular samples.}

Cylinder vessels placed in a rectangular plastic holder filled with the tap water were used. Cylinder vessels were filled with distilled water doped by several drops of magnetic liquid based on Dextran (polysaccharide).

For experimental evaluation of the mathematical modeling, two samples - circular vessels filled with doped water were placed to the centre of the holder. The central vessel was filled with tap water. Diameters of the vessels: $D_{1}=18, D_{2}=40, D_{3}=56 \mathrm{~mm}$, wall thickness: $1 \mathrm{~mm}$.

Resultant NMR image using GRE imaging sequence is depicted in Fig.6.

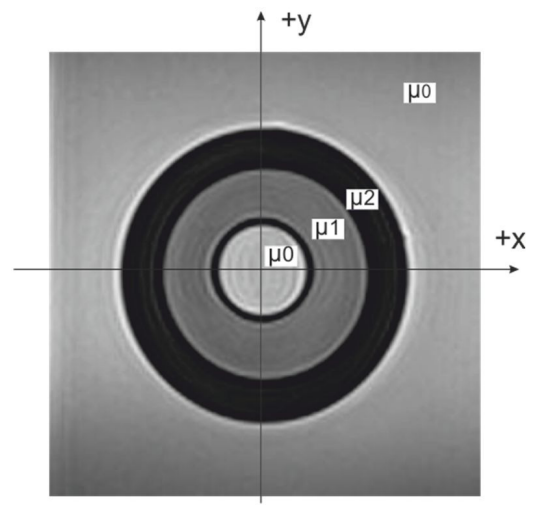

Fig.6. NMR image of liquid samples (three circular glass vessels filled with doped water in the rectangular boundary vessel - holder filled with tap water) using GRE imaging sequence: $T R=440 \mathrm{~ms}$, $\mathrm{TE}=10 \mathrm{~ms}$. Thickness of the imaged layer: $2 \mathrm{~mm}$.

\section{Weak magnetit solid circular samples.}

For sample positioning a plastic holder was constructed. The principal experimental arrangement is depicted in Fig.7.

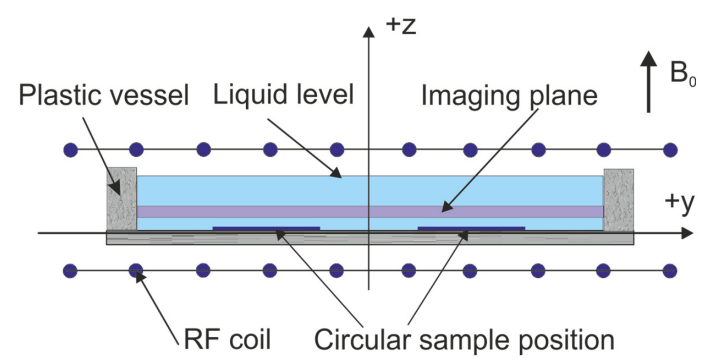

Fig.7. Sample positioning in the plastic holder. Static magnetic field of the imager $\mathbf{B}_{0}$ is perpendicular and RF field is parallel with the plane of the holder.

A radio frequency transducing coil (solenoid) together with the plastic was placed into the centre of the permanent magnet, perpendicular to the magnetic field $\left(\mathrm{B}_{0}\right)$ orientation.

The "gradient-echo" NMR sequences were selected for the measurements [8], [9]. A special feature of this sequence is its sensitivity to basic magnetic field inhomogeneities. The actual parameters used in presented experiments are as follows: RF pulse: $90^{\circ}$, repetition time: $\mathrm{TR}=800 \mathrm{~ms}$, echo time: $\mathrm{TE}=10 \mathrm{~ms}$, number of averages: 6 , max. measured matrix: 256 × 238, field of view: $140 \times 130$.
An NMR image of the magnetic annulus (cut from the data diskette) is depicted in Fig.8. The annulus was placed to the plastic holder and flooded by the water. The image represents magnetic field variations detected via water medium. The measurement was performed two times with different echo times TE. The Fourier transformed data matrices were ratio processed to prevent phase wrapping and to remove some distortions [14]. The magnetic field scale relates to the circular image of the sample.
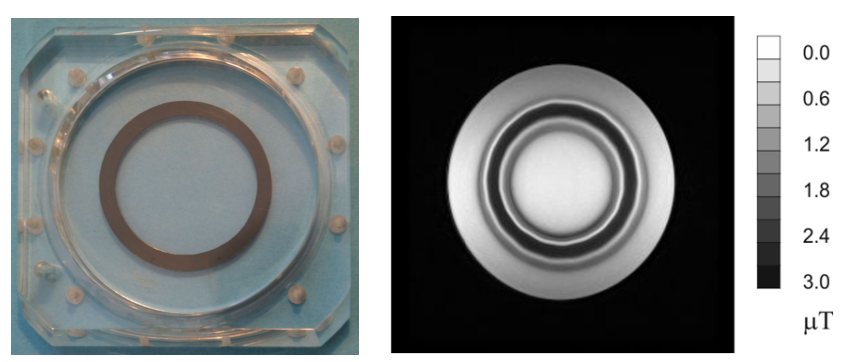

Fig.8. Left: Plastic holder used for sample positioning. Dish for the testing liquid. Circular sample dimensions: $\mathrm{D}_{1}=60 \mathrm{~mm}, \mathrm{D}_{2}=50 \mathrm{~mm}$. Right: Magnitude image of the magnetic annulus. Size of the measured matrix: $256 \times 256$.

Similar imaging sequences were used for imaging of commercial metal coins, see Fig.9. In the MR image an interesting structure is visible that illustrates the metal inhomogeneities inside the coins.
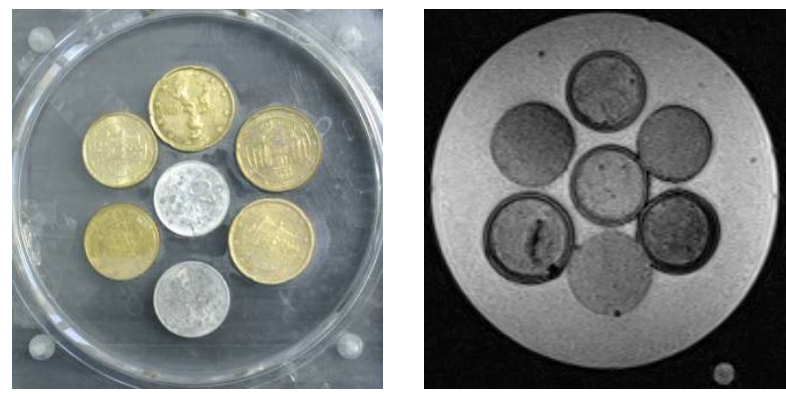

Fig.9. Examples of imaging of commercial coins. Left: Photo of 7 coins. Right: MR image, material inhomogeneities are clearly visible when GRE sequence was used.

For experimental verification the MRI 0.178 Tesla ESAOTE Opera imager (Esaote, Genoa, Italy) with vertical orientation of the basic magnetic field was used.

Several gradient-echo sequences were tested. It is evident, that every designed sequence is generating different image. We tried to find the best correlation between calculated results and resultant images.

\section{CONCLUSIONS}

The objective of this paper was to present a mathematical simulation of soft magnetic circular objects properties and using experimental magnetic resonance imaging methods for simple circular objects - vessels filled with doped water 
by very diluted magnetic liquids and magnetit solid circular samples using special plastic holder for sample positioning.

Mathematical analysis of circular objects, representing a shaped magnetic soft layer, showed theoretical possibilities to calculate magnetic field around any type of sample. Our mathematical models proved that it is possible to map the magnetic field variations - line of force - and to image the specific structures of selected samples placed into a special plastic holders. The calculations were performed with relative values of input quantities, permeabilities and dimensions. The mathematical model was described in the form of general formulas.

For experimental presentation a classical gradient-echo measuring sequences were used.

The resultant MR images are encircled by narrow stripes that optically extend the width of the sample. This phenomenon is typical for susceptibility imaging, when one needs to measure local magnetic field variations representing sample properties [11], [12], [13].

All experimental results are in relative good correlation with the mathematical simulations in spite of used comparative quantities. The proposed methods confirm the possible suitability of the proposed method for detection of weak magnetic materials in liquid or thin layer low magnetic objects using the MRI methods.

\section{ACKNOWLEDGMENT}

This work was supported by the Slovak Scientific Grant Agency VEGA 2/0013/14, within the project of the Slovak Research and Development Agency Nr. APVV-0431-12 and project of the University scientific park for biomedicine, Bratislava, ITMS: 26240220087.

\section{REFERENCES}

[1] Joy, M., Scott, G., Henkelman, M. (1989). In vivo imaging of applied electric currents by magnetic resonance imaging. Magnetic Resonance Imaging, 7 (1), 89-94.

[2] Callaghan, P.T., Stepisnik, J. (1995). Spatiallydistributed pulsed gradient spin echo NMR using single-wire proximity. Physical Review Letters, 75, 4532-4535.

[3] Sekino, M., Matsumoto, T., Yamaguchi, K., Iriguchi, N., Ueno, S. (2004). A method for NMR imaging of a magnetic field generated by electric current. IEEE Transactions on Magnetics, 40 (4), 2188-2190.
[4] Mikulka, J., Gescheidtova, E., Bartusek, K. (2012) Soft-tissues image processing: Comparison of traditional segmentation methods with 2D active contour methods. Measurement Science Review, 12 (4), 153-161.

[5] Strbak, O., Kopcansky, P., Timko, M., Frollo, I. (2013). Single biogenic magnetite nanoparticle physical characteristics. A biological impact study. IEEE Transactions on Magnetics, 49 (1), 457-462.

[6] Gogola, D., Štrbák, O., Krafčík, A., Škrátek, M., Frollo, I. (2015). Magnetic resonance imaging of the static magnetic field distortion caused by magnetic nanoparticles: Simulation and experimental verification. Journal of Magnetism and Magnetic Materials, 380, 261-265.

[7] Frollo, I., Andris, P., Gogola, D., Přibil, J., Valkovič, L., Szomolányi, P. (2012). Magnetic field variations near weak magnetic materials studied by magnetic resonance imaging techniques. IEEE Transactions on Magnetics, 48 (8), 2334-2339.

[8] Haacke, E.M., Brown, R.W., Thompson, M.R., Venkatesan, R. (1999). Magnetic Resonance Imaging: Physical Principles and Sequence Design (1st ed.). Wiley-Liss.

[9] Marcon, P., Bartusek, K., Dokoupil, Z., Gescheidtova, E. (2012). Diffusion MRI: Mitigation of magnetic field inhomogeneities. Measurement Science Review, 12 (5), 205-212.

[10] Henke, H. (2007). Elektromagnetic Fields, Theory and Using. Springer. (in German)

[11] Sun, Z.H.I., Guo, M., Vleugels, J., Van der Biest, O., Blanpain, B. (2013). Processing of non-ferromagnetic materials in strong static magnetic field. Current Opinion in Solid State and Materials Science, 17 (4), 193-201.

[12] Mueller, C., Scheinert, G., Uhlmann, F.H. (2003). Sensitivity estimation for magnetic devices using a double-layer model. International Journal of Applied Electromagnetics and Mechanics, 17 (1-3), 221-230.

[13] Jianming, J. (1999). Electromagnetic Analysis and Design in Magnetic Resonance Imaging. CRC Press.

[14] Andris, P., Frollo, I. (2011). Optimized measurement of magnetic field maps using nuclear magnetic resonance (NMR). Measurement Science and Technology, 22 (4), 045501.

Received September 11, 2015. Accepted December 2, 2015. 